\title{
Relation entre l'intensité de l'hydrothermalisme ancien ayant affecté les roches mères et l'actuelle défoliation et jaunissement des résineux adultes : exemple du Louschbach (Vosges)
}

\author{
MP Turpault, M Bonneau, D Gelhaye \\ INRA, centre de Nancy, équipe Biogéochimie des écosystèmes forestiers, \\ 54280 Champenoux, France
}

(Reçu le 8 juin 1994; accepté le 5 octobre 1994)

\begin{abstract}
Résumé - Le faible taux de saturation en cations basiques est un des facteurs qui prédisposent les peuplements forestiers au dépérissement. Ces sols pauvres sont souvent développés sur des roches pauvres qui fournissent peu de cations basiques en s'altérant. Dans le massif des Vosges, d'âge hercynien, l'hydrothermalisme est par endroit très intense et doit être pris en compte. II modifie la composition chimique et minéralogique des roches et donc leur potentiel nutritif. Au Louschbach, le granite du Valtin (à quartz, feldspath potassique, plagioclase, biotite et muscovite) est recoupé par un filon de quartz + hématite + (mica blanc). Autour de ce filon, se développe une auréole d'altération où le granite est transformé. Il est injecté par des veinules de quartz. Les biotites sont transformées en micas blancs, hématite et quartz. Les plagioclases sont transformés en micas blancs et quartz. Dans la zone très altérée près du filon, la quantité de calcium et de magnésium a diminué de plus de la moitié. Au tour de ce filon, la défoliation et le jaunissement des sapins et des épicéas sont mesurés sur des placettes de $2500 \mathrm{~m}^{2}$ en fonction du degré d'hydrothermalisme. Le dépérissement augmente nettement lorsque l'hydrothermalisme est intense. II est plus fort sur les épicéas. Concernant la roche mère, un autre facteur très important pour le dépérissement est la présence de dépôts glaciaires. Ils sont très hétérogènes et influencent le dépérissement dans les 2 sens selon leur nature. Le temps est un facteur important et les mesures montrent que la défoliation a doublé sur une placette témoin entre 1992 et 1994.
\end{abstract}

sapin = Abies alba Mill / épicéa = Picea abies Karst $/$ dépérissement $/$ hydrothermalisme $/$ Vosges

Summary - Relationships between the intensity of past hydrothermalism in the bedrock and present needle loss and yellowing of adult coniferous stands. Example of Louschbach (Vosges mountains). The low basic cation presence in the cationic exchange capacity of soils is one of the factors which favours forest decline. Such poor soils are often developed on poor rock which releases few basic cations during weathering. In the Vosges mountains dating from the Hercynian age, hydrother- 
mal alteration has been very intense in places and must be taken into consideration. This changes the chemical and mineralogical composition of the rocks, and consequently their nutritive potential. In the Louschbach site, the Valtin granite (quartz, $K$-feldspar, plagioclase, biotite and muscovite) is crosscut by a large quartz/hematite/white mica vein. This vein is surrounded by an alteration halo where the granite is transformed. It is interspersed by some quartz veins (thickest $<500 \mu \mathrm{m}$ ). Biotite has been transformed into white mica, hematite and quartz. Plagioclase has been transformed into white mica and quartz. In the strongly altered zone near the vein, the amount of calcium and magnesium decreases by more than $50 \%$. Around this large vein, the needle loss and yellowing of fir (Abies alba Mill) and spruce (Picea abies Karst) were measured on plots of $2500 \mathrm{~m}^{2}$ according to the hydrothermal alteration intensity. The decline clearly increases with the intensity of the hydrothermal alteration and is stronger in the spruces. As regards the parent material, another factor that is very important for decline is glacial sediment. This is heterogeneous and influences the decline in both directions depending on their composition. Time is an important factor and the measurements show that needle loss doubled between 1992 and 1994 on the control plot.

white fir = Abies alba Mill / spruce = Picea abies Karst $/$ forest decline $/$ hydrothermalism / Vosges

\section{INTRODUCTION}

Le dépérissement dans le massif des Vosges a été étudié depuis une dizaine d'années (Landmann, 1991 ; Bonneau et Landmann, 1993). De nombreux facteurs interviennent sur le dépérissement et sont classés en 3 groupes (Landmann, 1991). Les facteurs prédisposants sont la sylviculture, les caractéristiques physiques du sol, le climat rigoureux et les dépôts atmosphériques acides qui jouent essentiellement sur la masse foliaire. Le faible taux de saturation des sols en cations basiques et les dépôts acides sont des facteurs prédisposants intervenant surtout sur le jaunissement. Les facteurs déclenchants sont de nature climatique : les séries d'années sèches sont suivies par une accentuation du dépérissement (Becker, 1987 ; Levy et Becker, 1987). Les facteurs aggravants sont essentiellement biotiques (faune pathogène). Dans cet article, nous nous intéresserons indirectement à un des facteurs prédisposants qui est le faible taux de saturation des sols en cations basiques. Bonneau et Fichter (1991) ont montré que le dépérissement des forêts vosgiennes est souvent plus marqué sur les sols pauvres qui sont généralement développés sur roches mères pauvres en cations basiques. À l'opposé, l'augmentation artificielle de la quantité de cations basiques sur la capacité d'échange par fertilisation entraîne une diminution très nette de la défoliation et du jaunissement (Bonneau, 1993 ; Nys, 1991 ; Belkacem, 1993).

À l'intérieur même d'un massif géologique cristallophyllien, la composition chimique et minéralogique peut varier. En effet, en dehors des hétérogénéités souvent relativement faibles lors de la mise en place, les roches peuvent subir d'importantes modifications physiques et chimiques au cours de leur histoire. Les massifs cristallophylliens des Vosges, comme la plupart des massifs, sont en général affectés par des événements hydrothermaux (Pagel, 1981 ; Griffault, 1987) qui peuvent localement intensément modifier la roche mère. Le granite du Valtin, situé dans la partie centrale des Vosges est un bon exemple de ces variations chimiques car il est fortement hydrothermalisé à l'approche de sa limite orientale (Hameurt, 1967). De plus, le site du Louschbach, au nord de ce massif, a fait l'objet de recherches concernant le dépérissement depuis 1985. Un suivi des solutions du sol a été effectué entre 1988 et 1992 et a conduit à l'établissement de bilan de cations (Mohamed Ahamed, 1989, 1992 ; Mohamed Ahamed et al, 1993 ; Ranger et al, 1994). Des 
expériences de fertilisation ont été mises en place depuis 1985 (Bonneau et al, 1991 ; Bonneau, 1993) et une cartographie aérienne du dépérissement a été faite en 1990 par I'IGN (Rapport IGN, 1991 ; Bonneau et Fichter, 1991).

Une étude pétrographique et cristallochimique du granite sain et hydrothermalisé du Valtin sur le site du Louschbach est présentée dans la première partie de cet article. Ensuite, la relation entre le degré d'hydrothermalisme de la roche mère et la masse foliaire et le jaunissement est établie à partir de mesures sur 13 placettes forestières de peuplement adulte de sapin (Abies alba Mill) ou d'épicéa (Picea abies Karst). Le potentiel disponible de cations basiques ainsi que les autres facteurs mis en évidence au Louschbach sont discutés.

\section{CADRE GÉOLOGIQUE}

Le massif des Vosges peut être subdivisé en 2 parties suivant la nature et l'âge des terrains (fig 1):

- le socle cristallophyllien, en partie antévarisque, est constitué de gneiss et migma-

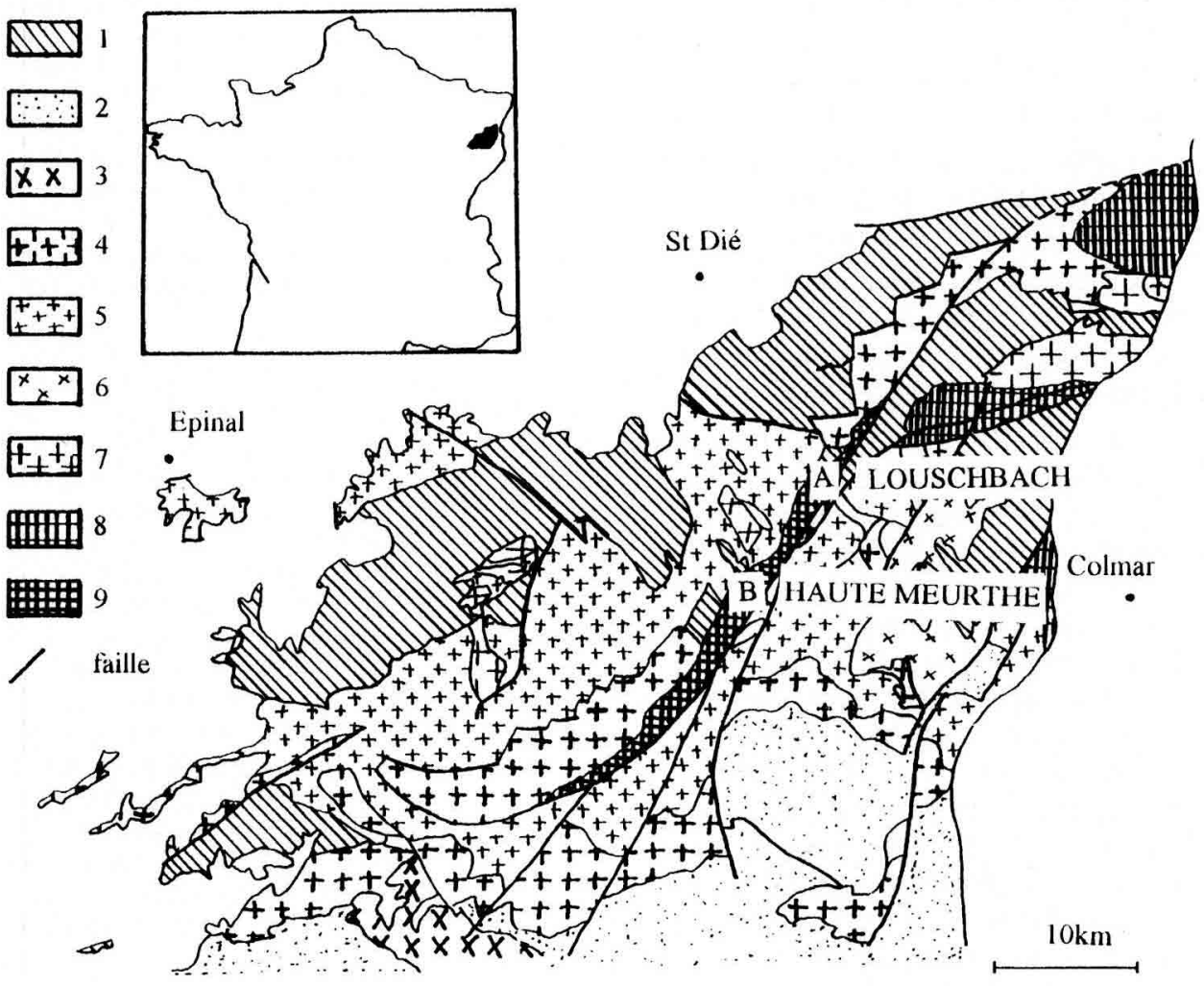

Fig 1. Carte géologique et structurale du socle des Vosges moyennes (d'après Fluck, 1980). A : mont Louschbach. B : site de la forêt de la haute Meurthe. 1 : gneiss et migmatite. 2 : Culm viséen. 3 : pluton des Ballons. 4 : granite du magmatisme des Crètes. $5:$ granite d'anatexie du socle gneissque ou granulitique. 6 : granite d'anatexie du Culm. $7:$ granite à mégacristaux. $8:$ leucogranite grossier. 9 : leucogranite grossier - granite du Valtin. 
tite plissés et métamorphisés qui sont injectés au cours des différentes phases de l'orogenèse hercynienne par des granitoïdes qui ont emprunté, pendant les phases de détente, les grands décrochements résultant des compressions (essentiellement $\mathrm{N}$ S) auxquelles était soumis ce socle.

- Des terrains volcano-sédimentaires du dévono-dinantien soumis durant la phase saalienne (Permien inférieur) à des mouvements cassants donnant naissance à des grabens qui sont comblés au Permo-trias.

Les gneiss sont d'origine sédimentaire (âge < 1000 MA : <Moldanubien) et ont subi 2 métamorphismes liés aux orogenèses moldanubienne et cadomienne (Hameurt, 1967).

Les granites mis en place durant la phase hercynienne sont de 2 types (Dahire, 1988):

- les granites d'anatexie crustale dont dérive la majeure partie des leucogranites à 2 micas : le Valtin, le Brézouard, le Bilstein ;

- les granites provenant de la différenciation des magmas basiques dont le massif des Ballons, des Crêtes, Thannenkirch, de Rombach Le Franc et le Châtenois.

Hameurt (1967) qui a notamment étudié le granite du Valtin donne la description suivante de ce massif. "ll a la forme d'une bande orientée $\mathrm{N}-\mathrm{NE}$, très allongée $(40 \mathrm{~km}$ de long et $2 \mathrm{~km}$ de large), s'effilant aux 2 extrémités, qui s'incurve au sud vers l'ouest. Il s'agit d'une lame inclinée vers l'est qui repose sur les formations cristallophylliennes dans lesquelles ce massif est intrusif et s'emboîte dans le granite des Crêtes qui possède le même gisement. Au toit, il bute contre la dislocation de Retournemer qui a contrôlé sa mise en place. Cette faille a rejoué de nombreuses fois. Le granite peut être fortement cataclasé et altéré hydrothermalement (silicifié et hématitisé) notamment à proximité de cette limite orientale. Un cortège filonien d'aplite et de microrganite accompagne la mise en place de ce granite. Ce massif s'apparente au granite du Brézouard et est rapporté à la phase asturienne (westphalien) de l'orogenèse de la chaîne varisque. La méthode de datation $\mathrm{Rb} / \mathrm{Sr}$ (Bonhomme, 1965) donne un âge compris entre 304 et $31410^{6}$ ans».

\section{MATÉRIELS ET MÉTHODES}

\section{Matériels}

Les difficultés concernant les échantillonnages destinés à mettre en évidence une relation entre l'hydrothermalisme des roches mères et le dépérissement actuel des arbres sont importantes. De nombreux paramètres intervenant sur le dépérissement, la mise en évidence de l'un d'entre eux implique un échantillonnage représentatif concernant la variation de ce seul facteur. Ceci est difficilement réalisable. II faut trouver une série de placettes représentatives peuplées d'arbres du même âge, ayant subi un type de sylviculture semblable, étant dans les mêmes conditions climatiques et topographiques où seuil le degré d'hydrothermalisme change. Les phénomènes hydrothermaux sont le plus souvent associés à l'ouverture de fissures dans la roche et donc localisés (quelques $\mathrm{cm}$ d'épaisseur autour de la fissure (Turpault et al, 1992) ou ils affectent massivement toute la roche (altération pervasive qui ne présente souvent pas de variation à l'échelle décimétrique). D'autre part, une mesure représentative du dépérissement sur un peuplement implique de compter au minimum 25 arbres. Le site du Louschbach est favorable pour établir cette relation car ce travail montre qu'à cet endroit le granite est recoupé par un filon hydrothermal à quartz + hématite + mica blanc de forte puissance (plusieurs dizaines de mètres) qui développe une auréole d'altération à ses épontes de plusieurs centaines de mètres. Dans une partie d'une éponte, non recoupée par d'autres filons, le granite présente un degré d'hydrothermalisme en fonction de la distance au filon de quartz. Les placettes étudiées sont principalement situées à cet endroit.

\section{Roche mère}

Les affleurements du granite du Valtin sont rares. Sur le site du Louschbach, les 2 affleurements 
observés sont échantillonnés. Ils présentent tous les 2 des traces d'altération supergène. L'échantillon GR7 est un granite non hydrothermalisé et l'échantillon BOD8 est hydrothermalisé. Les autres échantillons, prélevés dans des blocs décimétriques d'arène ou de sols des placettes sont hydrothermalisés (fig 2).

\section{Placettes forestières}

Afin de limiter l'influence des autres facteurs, les placettes choisies dans l'éponte du filon de quartz du Louschbach sont peuplées d'arbres adultes et situées à une altitude voisine (entre 1060 et $990 \mathrm{~m}$ ) avec des pentes relativement douces. Le dépérissement du sapin (Abies alba Mill) et de l'épicéa (Picea abies Karst) est testé en fonction du degré d'hydrothermalisme (fig 3 ). Sur le site du Louschbach, il n'a pas été possible de trouver des placettes équivalentes sur granite non hydrothermalisé (chablis, recouvrement glaciaire, altitude différente) ni de peuplement d'épicéa sur roche peu hydrothermalisé. Après une revue systématique des sites potentiels dans le massif du granite du Valtin situé au nord de la Vologne, une seule placette à épicéa et à sapin peu hydrothermalisé a été trouvée et comptée sur le site de la forêt de haute Meurthe près du village du Grand Valtin (fig 1).

\section{Méthodes}

Les parties des échantillons peu atteintes par l'altération supergène sont utilisées pour la réalisation de lames minces, la fabrication de poudre et pour le tri de certains minéraux après concassage de la roche. Les lames minces permettent une étude pétrographique sous microscope optique et des analyses ponctuelles à la microsonde (CAMEBAX MBX du service commun d'analyse de Nancy). Les erreurs analytiques à la microsonde sont de $\pm 1,5 \%$ pour $\mathrm{SiO}_{2}, \mathrm{Al}_{2} \mathrm{O}_{3}$, $\mathrm{MgO}$, de $\pm 1 \%$ pour $\mathrm{K}_{2} \mathrm{O}, \mathrm{CaO}, \mathrm{TiO}_{2}, \mathrm{MnO}, \mathrm{FeO}$ et de $\pm 3 \%$ pour $\mathrm{Na}_{2} \mathrm{O}$. Les résultats sont présentés sous forme de moyenne de 15 analyses minimum par type de minéral (en masse d'oxydes et en formule structurale). Le fer est calculé sous forme $\mathrm{Fe}^{2+}$. Les poudres sont utilisées pour l'analyse totale de l'échantillon. Une partie de la poudre est solubilisée à $1000^{\circ} \mathrm{C}$ avec du tétraborate de lithium et une analyse totale élémentaire est effectuée au spectromètre d'émission à arc pour les éléments $\mathrm{Si}, \mathrm{Al}, \mathrm{Fe}$,
$\mathrm{Mg}, \mathrm{Mn}$ et Ti, au spectromètre de flamme pour $\mathrm{Na}$ et $\mathrm{K}$ et au spectromètre à plasma pour le phosphore, le calcium et les éléments traces (Samuel et Rouault, 1983). L'autre partie de la poudre est utilisée afin de connaître la composition minéralogique à partir de la diffraction de rayons $X$ soit directement sur poudre ou sur lames orientées de fractions inférieures à $2 \mu \mathrm{m}$ après traitement par ultrason et décantation. Le diffractomètre utilisé est un Siemens 5000 équipé d'une anticathode cuivre.

Sur les placettes choisies, tous les arbres sont notés en pourcentage de perte d'aiguilles et en jaunissement avec une incertitude de plus ou moins $5 \%$. Les arbres morts ont reçu des notes de $100 \%$. Les résultats par placette comprenant au minimum 40 arbres sont la moyenne des notes des arbres de la placette. Afin de comparer les résultats du jaunissement avec ceux des années précédentes, 5 classes sont établies suivant le pourcentage de jaunissement (classe 0 : pas de jaunissement; classe 1:1 à 10\%; classe $2: 11$ à $25 \%$; classe $3: 26$ à $60 \%$, classe $4:>61 \%$ ).

\section{RÉSULTATS}

\section{Roche mère}

\section{Roche mère non hydrothermalisée}

L'échantillon GR7 est composé de quartz, plagioclase, fedlspath potassique (fréquemment du microcline), biotite, quelques muscovites et des minéraux accessoires (zircon, apatite). Quelques rares micas blancs de petite taille se sont formés dans les feldspaths. Les recristallisations de feldspaths à leur périphérie sont fréquentes. Des micas blancs très fins pseudomorphosent des cristaux de forme globuleuse qui sont vraisemblablement des corditérites. La précipitation de quartz au joint de grain des minéraux est vraisemblablement en relation avec la mise en place du filon de quartz.

L'analyse de GR7 est proche des analyses de la littérature (tableau I). Elle présente cependant une teneur plus élevée en 


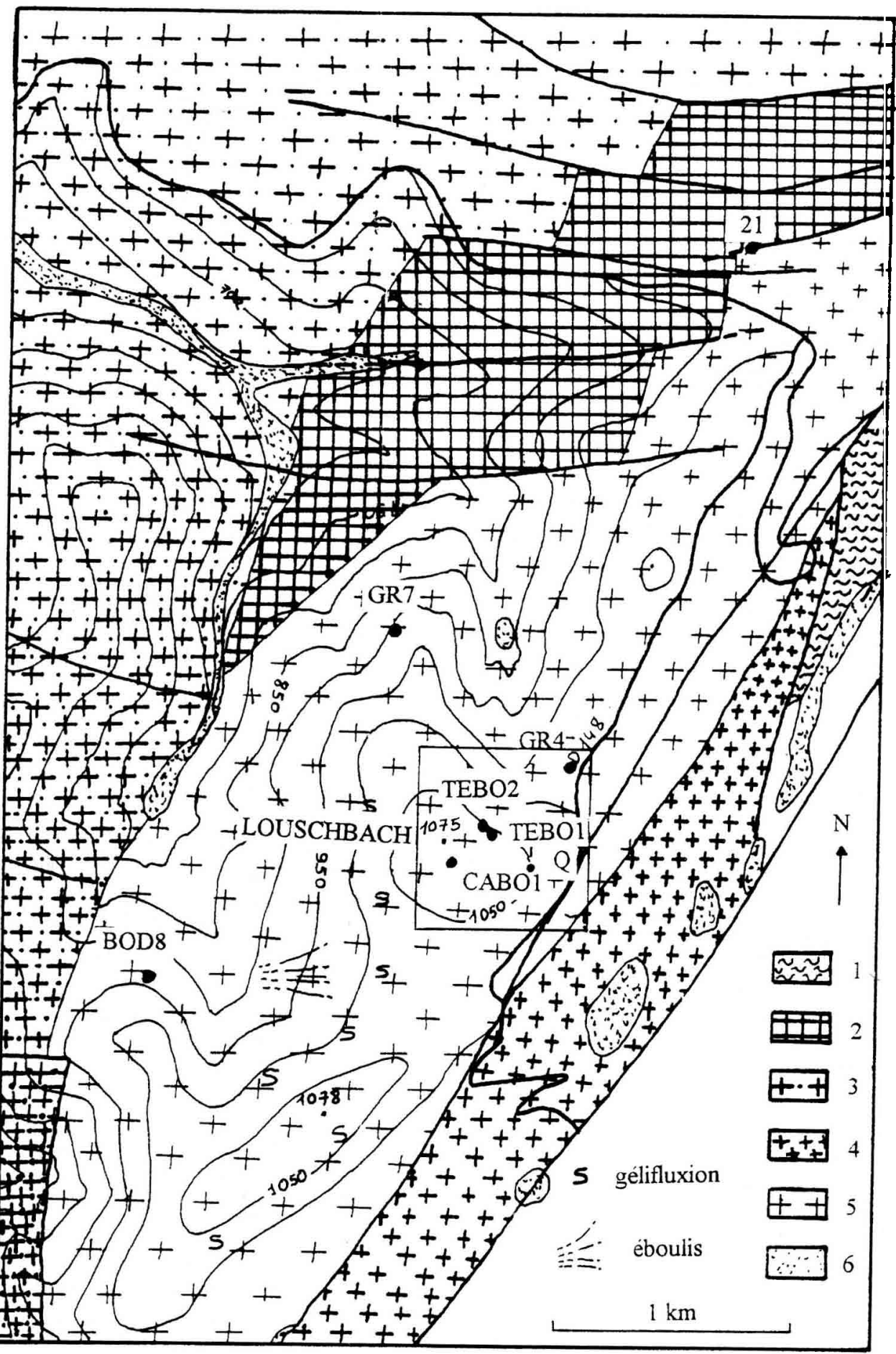




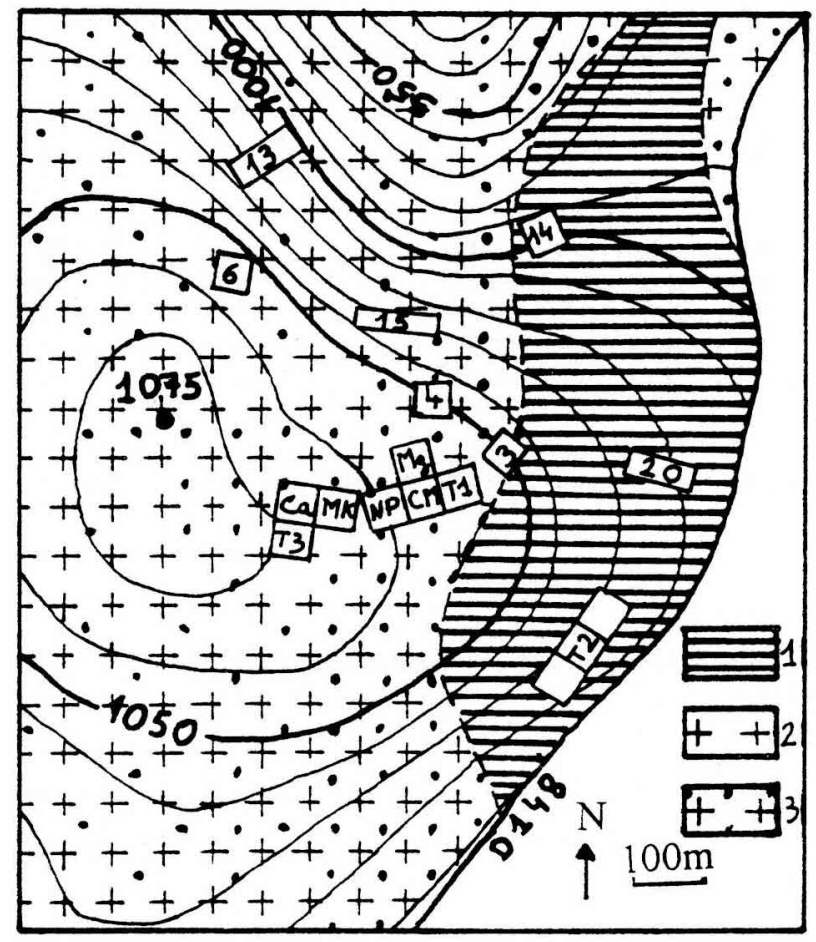

Fig 3. Site du Louschbach Cartographie du filon de quartz (1) et du degré d'hydrothermalisme du granite du Valtin (2: granite non hydrothermalisé ; 3 : granite fortement hydrothermalisé). Positionnement des placettes mesurées (numérotées) et des placettes fertilisées avec différents éléments ( $\mathrm{Ca} ; \mathrm{MK}=\mathrm{Mg}+\mathrm{K}$; $\mathrm{NP}=\mathrm{N}+\mathrm{P}+\mathrm{K}+\mathrm{Ca}+\mathrm{Mg}$ $\mathrm{CM}=\mathrm{Ca}+\mathrm{Mg}$ ). silice et des teneurs moins élevées des autres oxydes. Cette teneur élevée en silice est due au quartz précipité entre les joints de grain. Les analyses chimiques des minéraux effectuées sur les échantillons GR7 sont données dans le tableau II. Microcline et orthose se distinguent par leur teneur en sodium. Le plagioclase est une albite avec $9 \%$ d'anorthite $(\mathrm{Ca} / \mathrm{Ca}+\mathrm{Na}=0,09$; rapport molaire). La biotite est pauvre en magnésium $(\mathrm{Mg} / \mathrm{Fe}+\mathrm{Mg}=0,2$; rapport molaire). La muscovite primaire contient du sodium $(\mathrm{Na} / \mathrm{Na}+\mathrm{K}=0,09$; rapport molaire). Le mica blanc vraisemblablement deutérique, précipité dans le feldspath potassique, a la même composition que la muscovite.

À partir des analyses des minéraux et de l'analyse totale de l'échantillon GR7, les quantités de minéraux ont pu être établies. Cette détermination est basée sur un système de 4 équations (4 éléments chimiques: $\mathrm{Na}, \mathrm{K}, \mathrm{Al}$ et $\mathrm{Mg}$ ) à 4 inconnues (pourcentage de feldspath potassique avec un quart de microcline et 3 quarts d'orthose, de plagioclase, de muscovite et de biotite). Le pourcentage d'apatite est déduit de la teneur en phosphore.

Fig 2. Carte géologique du site du Louschbach (d'après la carte géologique au 1/50 000) et positionnement des échantillons prélevés pour l'étude de la roche mère. 1 : groupe des gneiss de SainteMaris-aux-Mines - granulite claire. 2 : groupe des gneiss de la Croix-aux-Mines - gneiss perlé à cordiérite. 3 : granito-gneiss. $4:$ granite des Crètes. $5:$ granite du Valtin. 6 : Dépôts fluvio-glaciaires et formations glaciaires. 
Tableau I. Composition chimique (en $\mathrm{g}$ d'oxydes dans $100 \mathrm{~g}$ de roche) du granite du Valtin non hydrothermalisé $(n \mathrm{H})$ et hydrothermalisé à différentes intensités $\left(\mathrm{H}^{+}, \mathrm{H}^{++}, \mathrm{H}^{+++}\right)$.

$\begin{array}{lllllllllllllllllll}\text { Échantillon } \mathrm{H} & \mathrm{SiO} & \mathrm{Al}_{2} \mathrm{O}_{3} & \mathrm{MgO} & \mathrm{CaO} & \mathrm{Fe}_{2} \mathrm{O}_{3} & \mathrm{TiO}_{2} & \mathrm{P}_{2} \mathrm{O}_{5} & \mathrm{Na}_{2} \mathrm{O} & \mathrm{K}_{2} \mathrm{O} & \text { PAF } & \text { Total }\end{array}$

$\begin{array}{llllllllllllc}\text { GR7 } & \mathrm{nH} & 77,6 & 12,7 & 0,22 & 0,51 & 0,9 & 0,11 & 0,17 & 2,69 & 4,83 & 0,53 & 100,25 \\ \text { Moy }^{\mathrm{a}} & \mathrm{nH} & 74,39 & 13,77 & 0,22 & 0,62 & 1,47 & 0,13 & \text { nd } & 3,57 & 4,67 & 0,93 & 99,77 \\ \text { TEBO2 } & \mathrm{H}^{+} & 76,8 & 12,3 & 0,44 & 0,3 & 2,2 & 0,25 & 0,11 & 2,37 & 5,42 & 0,71 & 100,90 \\ \text { BOD8 } & \mathrm{H}^{++} & 73,41 & 14,06 & 0,13 & 0,32 & 0,8 & 0,1 & 0,07 & 3,48 & 6,18 & \text { nd } & \text { nd } \\ \text { TEBO1 } & \mathrm{H}^{+++} & 82,00 & 10,20 & 0,09 & 0,14 & 0,8 & 0,11 & <0,1 & 1,94 & 4,70 & 0,39 & 100,37\end{array}$

a Analyse moyenne dans Hameurt (1967); nd : non déterminé ; PAF : perte au feu $1000^{\circ} \mathrm{C}$.

Tableau II. Composition chimique (en \% g d'oxydes) et formule structurale des minéraux du granite du Valtin non hydrothermalisé (affleurement GR7).

\begin{tabular}{|c|c|c|c|c|c|c|}
\hline $\begin{array}{l}\text { Analyses } \\
\text { moyennes } \\
\text { minéraux GR7 }\end{array}$ & Orthose & Microcline & Plagioclase & Biotite & Muscovite & $\begin{array}{c}\text { Mica blanc } \\
\text { précipité dans } \\
\text { feldspath } K\end{array}$ \\
\hline- & $\ldots$ & $\cdots \cdots$ & $\cdots$ & $\cdots \quad-$ & $-\ldots$. & $\ldots \ldots$ \\
\hline $\mathrm{SiO} 2$ & 65,41 & 65,1 & 66,06 & 35,25 & 46,25 & 46,36 \\
\hline $\mathrm{Al} 2 \mathrm{O} 3$ & 19,05 & 18,8 & 21,23 & 20,65 & 35,04 & 35,03 \\
\hline $\mathrm{FeO}$ & 0 & 0 & 0 & 22,24 & 1,81 & 1,84 \\
\hline $\mathrm{MgO}$ & 0 & 0 & 0 & 3,16 & 0,79 & 0,75 \\
\hline $\mathrm{MnO}$ & 0 & 0 & 0 & 0,94 & 0,09 & 0,08 \\
\hline TiO2 & 0 & 0 & 0 & 2,89 & 0,19 & 0,15 \\
\hline $\mathrm{Na} 2 \mathrm{O}$ & 0,78 & 1,84 & 10,27 & 0,05 & 0,66 & 0,48 \\
\hline K2O & 15,32 & 14,31 & 0,42 & 9,25 & 10,07 & 10,46 \\
\hline $\mathrm{CaO}$ & 0 & 0 & 1,74 & 0 & 0 & 0 \\
\hline Total & 100,6 & 100,1 & 99,73 & 94,43 & 94,90 & 95,13 \\
\hline Base & 8 & 8 & 8 & 11 & 11 & 11 \\
\hline Al total & 1,02 & 1,02 & 1,10 & 1,90 & 2,76 & 2,76 \\
\hline $\mathrm{Si}$ & 2,99 & 2,99 & 2,91 & 2,74 & 3,09 & 3,10 \\
\hline Al IV & & & & 1,26 & 0,91 & 0,90 \\
\hline Al VI & & & & 0,64 & 1,85 & 1,86 \\
\hline $\mathrm{Fe}$ & 0 & 0 & 0 & 1,45 & 0,10 & 0,10 \\
\hline $\mathrm{Mg}$ & 0 & 0 & 0 & 0,36 & 0,08 & 0,07 \\
\hline $\mathrm{Ti}$ & 0 & 0 & 0 & 0,17 & 0,01 & 0,01 \\
\hline $\mathrm{Mn}$ & 0 & 0 & 0 & 0,06 & 0 & 0,01 \\
\hline OCT & & & & 2,68 & 2,05 & 2,04 \\
\hline $\mathrm{K}$ & 0,89 & 0,84 & 0,02 & 0,92 & 0,86 & 0,89 \\
\hline $\mathrm{Na}$ & 0,07 & 0,16 & 0,88 & 0,08 & 0,08 & 0,06 \\
\hline $\mathrm{Ca}$ & 0 & 0 & 0,08 & 0 & 0 & 0 \\
\hline
\end{tabular}

AlIV : aluminium tétraédrique; ALVI : aluminium octaédrique ; OCT : nombre de cations dans l'octaèdre. 
Nous trouvons $42 \%$ de quartz, $24 \%$ de feldspath $\mathrm{K}, 23 \%$ de plagioclase, $5,8 \%$ de muscovite, $5,5 \%$ de biotite et $0,4 \%$ d'apatite (tableau III). Hameurt (1967) donne une composition minéralogique d'un échantillon prélevé au sud du massif (La Bresse) qui est différente : $36 \%$ de quartz, $33 \%$ feldspath potassique, $22 \%$ de plagioclase, $3,5 \%$ de biotite, $2,5 \%$ de muscovite et $2,5 \%$ de zircon, apatite, chlorite, pinite et hématite).

\section{Roche mère hydrothermalisée}

L'hydrothermalisme au Louschbach se traduit par un filon à quartz + hématite \pm illite de puissance décimétrique, par des veinules de quelques centimètres d'épaisseur et par une transformation sélective du granite aux épontes de ces drains.

\section{Le filon décimétrique à quartz + hématite + mica blanc}

Quelques affleurements alignés de quartz sont présents sur la bordure ouest du filon au point $Q$ (fig 2). L'orientation de ces affleurements est $\mathrm{S}-\mathrm{SW}\left(20^{\circ} \mathrm{W}\right)$. Le pendage n'a pas pu être mesuré. Le reste du filon est cartographié à partir des pierres volantes. Sa puissance affleurante est probablement surestimée du fait de la pente du terrain qui entraîne les pierres vers le bas.
Les affleurements en place sont constitués principalement de quartz laiteux recoupé par un réseau anastomosé de veinules d'hématite et des veinules à quartz + hématite rectilignes. Sur les pierres volantes (non en place), des brèches de quartz laiteux anguleux celés par un ciment d'hématite sont observées, ainsi que la présence de veinules à quartz + mica blanc donnant une couleur verdâtre.

$A$ priori, il est impossible, à partir de telles observations, de déterminer la quantité de chaque minéral du filon et leur répartition. Par exemple, le filon contient peut-être une quantité non négligeable de mica blanc qui n'est plus visible en surface car elle est reprise par l'altération supergène.

L'observation rapide du filon montre qu'il a subi plusieurs épisodes de fracturationsdépôts successifs. Le filon de quartz laiteux original est recoupé par des veines à hématite, à hématite-quartz et à quartz-mica blanc.

\section{Auréole d'altération autour du filon de quartz}

Autour du grand filon de quartz, l'auréole d'altération est d'au moins $500 \mathrm{~m}$. Dans une direction perpendiculaire au filon, entre les courbes de niveau 1050 et $1000 \mathrm{~m}$ (fig 3), le granite n'est pas influencé par d'autres grands filons et présente un gradient d'altération en

Tableau III. Pourcentage (en masse) des minéraux dans le granite sain (LB et GR7) et le granite très hydrothermalisé (TEBO1) du site du Louschbach.

\begin{tabular}{lccccccc}
\hline & Quartz & FeldspathK & Plagioclase & Biotite & Mica blanc & Apatite & Hématite \\
& & & & & & & \\
LB a & 36 & 33 & 22 & 3,5 & 2,5 & nd & 0 \\
GR7 & 42 & 24 & 23 & 5,5 & 5,8 & 0,4 & 0 \\
TEBO1 & 52 & 24 & 16 & 0 & 8 & 0,3 & 0,7
\end{tabular}

a Composition minéralogique donnée dans Hameurt (1967). 
fonction de la distance au filon. Les placettes où sont faites les mesures de masse foliaire et de jaunissement sont choisies principalement dans cette zone (fig 3), La transformation est de plus en plus intense à proximité du filon. Afin d'étudier cette zone, 2 pierres volantes décimétriques sont choisies. L'une est très altérée (TEBO1) et aucune biotite n'est visible. L'autre est moyennement altérée et quelques biotites non transformées ont pu être observées (TEBO2). L'échantillon BOD8 prélevé en place en dehors de l'éponte de ce filon de quartz présente un degré d'hydrothermalisme intermédiaire entre TEBO1 et TEBO2. Des biotites partiellement transformées sont observées.

\section{Échantillon très altéré}

La biotite a complètement disparu. Elle est remplacée par de l'hématite et du mica blanc et parfois du quartz. Le plagioclase est partiellement transformé en micas blancs. Son pourcentage d'albite augmente à $99 \%$ $(\mathrm{Ca} / \mathrm{Ca}+\mathrm{Na}=0,01)$. Du quartz secondaire précipite à de nombreux joints de grains. De plus quelques veinules de quartz recoupent le granite. Ces transformations se traduisent sur l'analyse totale par une augmentation de $\mathrm{SiO}_{2}$ et une diminution de tous les autres oxydes. Le magnésium a diminué de plus de moitié et le calcium de plus de $70 \%$ par rapport à la roche mère saine. La biotite contenant $3,16 \%$ de $\mathrm{MgO}$ est remplacée partiellement par un mica blanc à 1,1\% de $\mathrm{MgO}$. L'albite saine est remplacée par des minéraux non calciques (mica blanc, quartz) et par une albite moins calcique. Le potassium est à peu près maintenu car des micas blancs avec $10,5 \%$ de $\mathrm{K}_{2} \mathrm{O}$ remplacent partiellement la biotite $\left(\mathrm{K}_{2} \mathrm{O}=9,25 \%\right)$ et précipitent dans les plagioclases et parfois dans les feldspaths potassiques. Le calcul du pourcentage de minéraux dans la roche à partir d'une norme confirme ces résultats : $52 \%$ de quartz, $16 \%$ de plagioclase, $24 \%$ de feldspath potassique, $8 \%$ de micas blancs, $0 \%$ de biotite, $0,7 \%$ d'hématite et $0,3 \%$ d'apatite (tableau III).

\section{Échantillons moyennement altérés}

L'échantillon TEBO2 présente des biotites saines et des biotites partiellement transformées en hématite et micas blancs. Les plagioclases sont partiellement transformés en micas blancs. Du quartz précipite également aux joints de grains des minéraux. À cette altération hydrothermale, se superpose une altération supergène. Cette altération se traduit par des fissures tapissées d'hydroxydes de fer. Le long de ces fissures les biotites sont partiellement transformées en kaolinite. La présence de la kaolinite est confirmée par la diffraction de rayons $X$. Au centre des plagioclases, les zones transformées partiellement en micas blancs sont imprégnées d'hydroxydes de fer. L'échantillon TEBO2 est donc le résultat de 2 transformations peu intenses : hydrothermale et supergène. L'altération supergène se traduit notamment par une augmentation de la teneur en magnésium et en fer. Cet échantillon ne peut donc pas être pris comme référence pour déterminer la quantité de cations basiques en fonction du degré d'hydrothermalisme.

En revanche, l'échantillon BOD8, bien que prélevé en dehors de l'éponte directe du filon de quartz mais en place, est peu affecté par l'altération supergène. II présente par ailleurs un degré d'altération hydrothermale plus important que TEBO2. En ce qui concerne le calcium et le magnésium (tableau III), il est effectivement intermédiaire entre l'échantillon très hydrothermalisé (TEBO1) et l'échantillon sain (GR7).

\section{Les veinules centimétriques}

Les échantillons prélevés dans l'éponte proche du filon sont recoupés par de nombreuses veinules (GR4, et dans une 
moindre mesure TEBO1). Des veines centimétriques à quartz seul sont recoupées par des veines millimétriques à quartz, à quartz-hématite ou par de très fines veines hétérogènes dont le remplissage dépend du minéral traversé. Certaines veines peuvent être reprises par de petites chlorites en accordéons. L'auréole d'altération de ces veines est inférieure à $1 \mathrm{~cm}$ et dépend de la veinule. Par exemple, les biotites peuvent être chloritisées autour de la veinule reprise par les chlorites. Cette altération observée une seule fois est négligeable à l'échelle de la placette forestière.

Remarquons que, sur le site du Grand Valtin (forêt domaniale de haute Meurthe, parcelle 17), le granite sain est recoupé par des veines centimétriques à millimétriques qui développent une auréole d'altération de quelques centimètres et où les biotites sont partiellement transformées. La densité des veines étant peu importante, le granite est qualifié de peu hydrothermalisé.

\section{Zone très altérée située au niveau de la placette $\mathrm{Ca}$}

Une zone, située au niveau de la placette $\mathrm{Ca}$ (fig 3), est également très altérée sans qu'aucun filon ne puisse être mis en évidence. Le granite est imprégné par du quartz, de l'hématite et du mica blanc. Les grains du granite sont complètement dissociés : il s'agit par endroit d'une brèche à quartz. Dans ces espaces cristallise du quartz automorphe sous forme géodique. En bordure, des quartz de petite taille précipitent puis viennent des quartz automorphes de grande dimension (du millimètre au centimètre). Des micas blancs peuvent précipiter par endroit au centre de ces veines. L'ensemble de la roche est imprégnée d'hématite. Les biotites sont entièrement transformées en hématite et micas blancs. Les plagioclases sont partiellement transformés en micas blancs.

\section{Relation entre le degré d'hydrothermalisme de la roche mère, la masse foliaire et la couleur}

Le tableau IV et la figure 4 montrent que le jaunissement et la défoliation augmentent avec le degré d'hydrothermalisme du granite. L'augmentation est plus marquée pour l'épicéa qui est en général plus dépérissant. Sur granite très hydrothermalisé, le jaunis-

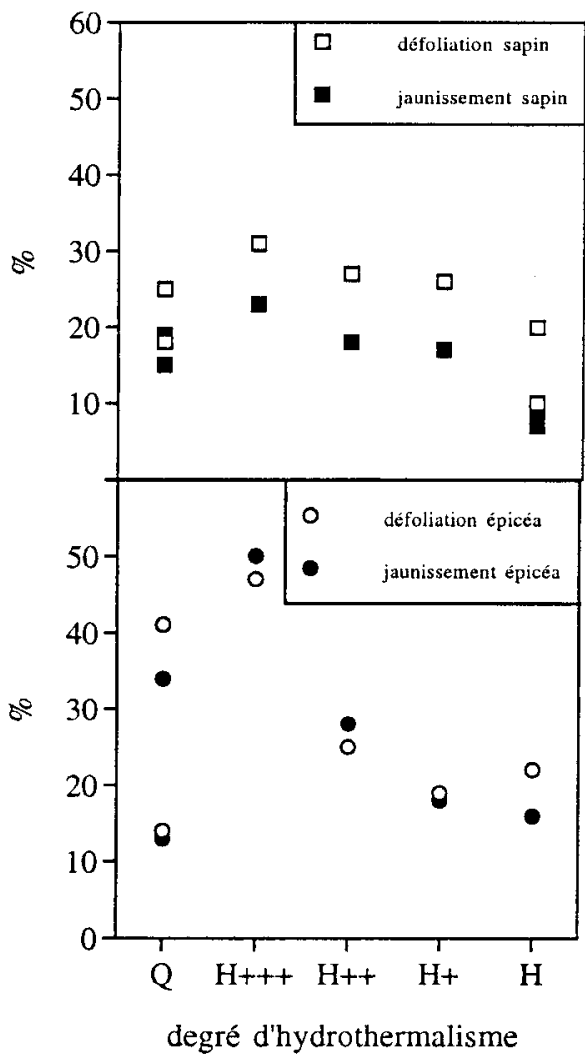

Fig 4. Pourcentage de défoliation (figurés vides) et d'aiguilles jaunies (figurés pleins) a) du sapin et b) de l'épicéa en fonction du degré d'hydrothermalisme du granite $(\mathrm{H}+++$ : granite très hydrothermalisé ; $\mathrm{H}_{+}$: granite légèrement hydrothermalisé ; $Q$ : filon de quartz + hematite + micas blancs). 
Tableau IV. Composition chimique (en \% g d'oxydes) et formule structurale des minéraux du granite du Valtin très hydrothermalisé (échantillon TEBO1).

\begin{tabular}{|c|c|c|c|c|c|c|c|}
\hline $\begin{array}{l}\text { Analyses } \\
\text { des } \\
\text { minéraux } \\
\text { de la } \\
\text { roche mère }\end{array}$ & Orthose & Microcline & Plagioclase & $\begin{array}{l}\text { Muscovite } \\
\text { hématisée }\end{array}$ & $\begin{array}{c}\text { Mica blanc } \\
\text { précipité } \\
\text { dans } \\
\text { plagioclase }\end{array}$ & $\begin{array}{c}\text { Mica blanc } \\
\text { précipité } \\
\text { dans } \\
\text { orthose }\end{array}$ & $\begin{array}{c}\text { Mica blanc } \\
\text { précipité } \\
\text { dans } \\
\text { muscovite }\end{array}$ \\
\hline $\mathrm{SiO} 2$ & 64,41 & 64,51 & 68,11 & 48,21 & 47,02 & 47,16 & 46,40 \\
\hline $\mathrm{Al} 2 \mathrm{O} 3$ & 18,13 & 18,35 & 19,86 & 31,38 & 34,75 & 33,09 & 32,46 \\
\hline $\mathrm{FeO}$ & 0 & 0 & 0 & 1,95 & 1,06 & 1,48 & 1,79 \\
\hline $\mathrm{MgO}$ & 0 & 0 & 0 & 1,10 & 0,57 & 0,80 & 1,00 \\
\hline MnO & 0 & 0 & 0 & 0 & 0,03 & 0,07 & 0 \\
\hline $\mathrm{TiO} 2$ & 0 & 0 & 0 & 0,34 & 0,03 & 0 & 0,71 \\
\hline $\mathrm{Na} 2 \mathrm{O}$ & 0,60 & 0,77 & 11,31 & 0,03 & 0,11 & 0 & 0,32 \\
\hline $\mathrm{K} 2 \mathrm{O}$ & 15,69 & 15,74 & 0,04 & 10,46 & 10,20 & 10,71 & 10,60 \\
\hline $\mathrm{CaO}$ & 0 & 0 & 0,30 & 0 & 0 & 0 & 0 \\
\hline Total & 99,04 & 99,36 & 99,62 & 93,47 & 93,79 & 93,30 & 93,31 \\
\hline Base & 8 & 8 & 8 & 11 & 11 & 11 & 11 \\
\hline Al total & 1,00 & 1,00 & 1,03 & 2,51 & 2,75 & 2,65 & 2,61 \\
\hline $\mathrm{Si}$ & 3,00 & 3,00 & 2,98 & 3,27 & 3,16 & 3,20 & 3,16 \\
\hline Al IV & & & & 0,73 & 0,84 & 0,80 & 0,84 \\
\hline $\mathrm{Al} \mathrm{VI}$ & & & & 1,77 & 1,90 & 1,85 & 1,77 \\
\hline $\mathrm{Fe}$ & 0 & 0 & 0 & 0,11 & 0,06 & 0,08 & 0,10 \\
\hline $\mathrm{Mg}$ & 0 & 0 & 0 & 0,111 & 0,06 & 0,08 & 0,10 \\
\hline $\mathrm{Ti}$ & 0 & 0 & 0 & 0,02 & 0 & 0 & 0,04 \\
\hline $\mathrm{Mn}$ & 0 & 0 & 0 & 0 & 0 & 0 & 0 \\
\hline OCT & & & & 2,01 & 2,02 & 2,01 & 2,01 \\
\hline K & 0,95 & 0,93 & 0 & 0,90 & 0,87 & 0,93 & 0,92 \\
\hline $\mathrm{Na}$ & 0,05 & 0,07 & 0,96 & 0 & 0,01 & 0 & 0,04 \\
\hline $\mathrm{Ca}$ & 0 & 0 & 0,01 & 0 & 0 & 0 & 0 \\
\hline
\end{tabular}

Al IV : aluminium tétraédrique ; Al VI : aluminium octaédrique ; OCT : nombre de cations dans l'octaèdre.

sement et la défoliation de l'épicéa atteignent $50 \%$. Pour l'épicéa, les courbes de jaunissement et de défoliation sont proches et augmentent fortement dans la zone très hydrothermalisée. La défoliation du sapin est plus marquée que son jaunissement. La défoliation des résineux de la placette 17 dans la forêt domaniale de la haute Meurthe sur granite peu hydrothermalisé est importante sans que, a priori, le degré d'hydrothermalisme soit une explication. Sur ce granite peu hydrothermalisé, elle est 2 fois plus élevée pour les sapins que sur le site du Louschbach alors que le jaunissement est semblable.

L'altitude n'est pas toujours constante et semble intervenir. Les points au-dessous des courbes correspondent à des altitudes inférieures à $1050 \mathrm{~m}$.

Remarquons que, sur le filon, l'intensité du dépérissement est variable. En général sur les placettes choisies, il est moins important que sur le granite très hydrothermalisé. Comme il n'est pas possible de cartographier de manière précise les minéraux de 
Tableau V. Mesures du pourcentage de défoliation et de jaunissement sur 12 placettes de sapins et d'épicéas en fonction du degré d'hydrothermalisme.

\begin{tabular}{|c|c|c|c|c|c|c|c|c|c|c|c|c|}
\hline \multirow[t]{2}{*}{ Placette } & \multirow{2}{*}{$\begin{array}{l}\text { Altitude } \\
\text { (en } m \text { ) }\end{array}$} & \multirow{2}{*}{$\begin{array}{c}\text { Nombre d'arbres } \\
\text { mesurés }\end{array}$} & \multicolumn{5}{|c|}{ Défoliation } & \multicolumn{5}{|c|}{ Jaunissement } \\
\hline & & & $Q$ & $\mathrm{H}^{+++}$ & $\mathrm{H}^{++}$ & $\mathrm{H}^{+}$ & $H$ & $Q$ & $\mathrm{H}^{+++}$ & $\mathrm{H}^{++}$ & $H^{+}$ & $H$ \\
\hline \multicolumn{13}{|l|}{ Sapin } \\
\hline 14 & 990 & 37 & 25 & & & & & 19 & & & & \\
\hline 3 & 1050 & 26 & 18 & & & & & 15 & & & & \\
\hline 4 & 1050 & 32 & & 31 & & & & & 23 & & & \\
\hline 15 & 1030 & 57 & & & 27 & & & & & 18 & & \\
\hline 6 & 1050 & 42 & & & & 26 & & & & & 17 & \\
\hline 13 & 1020 & 37 & & & & & 10 & & & & & 7 \\
\hline 17 & 1050 & 47 & & & & & 20 & & & & & 8 \\
\hline \multicolumn{13}{|l|}{ Épicéa } \\
\hline $\mathrm{T} 2$ & 1030 & 25 & 14 & & & & & 13 & & & & \\
\hline 20 & 1020 & 59 & 41 & & & & & 34 & & & & \\
\hline T1 & 1055 & 45 & & 47 & & & & & 50 & & & \\
\hline 15 & 1030 & 16 & & & 25 & & & & & 28 & & \\
\hline T3 & 1065 & 25 & & & & 19 & & & & & 18 & \\
\hline 17 & 1050 & 43 & & & & & 22 & & & & & 16 \\
\hline
\end{tabular}

$H$ : sur granite très peu hydrothermalisé qui présente quelques biotites hématitisées. $Q$ : sur filon de quartz. $H^{+++}$: sur granite très hydrothermalisé correspondant à l'échantillon TEBO1.

remplissage (par exemple le mica blanc), aucune correspondance entre la richesse en cations basiques de la roche mère et le dépérissement ne peut être faite au niveau du filon.

\section{DISCUSSION}

\section{Relation entre le degré d'hydrothermalisme et la quantité de cations basiques de la roche mère}

Le type d'hydrothermalisme qui a affecté le granite du Valtin au Louschbach diminue la quantité de cations basiques de cette roche qui est déjà très pauvre en ces éléments $(-50 \%$ pour $\mathrm{CaO}$ et $-70 \%$ pour $\mathrm{MgO})$. L'hydrothermalisme se traduit par l'injection de veinules essentiellement à quartz, ce qui a pour conséquences l'augmentation de $\mathrm{SiO}_{2}$ et la transformation des minéraux primaires. La transformation de la biotite en hématite + mica blanc + (quartz) induit une diminution de magnésium. La transformation du plagioclase en micas blancs et en plagioclase presque exclusivement sodique contribue à la diminution de calcium. La quantité d'apatite, phosphate de calcium, a également diminué dans le granite hydrothermalisé. Le potassium reste stable ou augmente (précipitation des micas blancs). Non seulement I'hydrothermalisme modifie la quantité globale d'éléments, mais aussi leur répartition dans les minéraux. Dans le granite très hydrothermalisé, le calcium est presque exclusivement dans l'apatite, le magnésium se trouve uniquement dans les micas blancs (muscovite primaire et micas 
blancs hydrothermaux) et le potassium est contenu dans les micas blancs et les feldspaths. De plus, I'hydrothermalisme modifie les propriétés physiques du granite. Les plagioclases et les biotites altérés présentent une porosité généralement plus importante. Cependant, l'injection de quartz, minéral très résistant à l'altération, en veinules ou aux joints des minéraux, confère au granite une moins grande altérabilité.

\section{Relation potentiel d'éléments nutritifs-dépérissement}

À l'échelle de la placette forestière $(50 \times 50$ $\mathrm{m})$, ce travail a clairement démontré la relation qui existe entre la quantité de cations basiques $(\mathrm{Ca}, \mathrm{Mg})$ dans la roche mère et le dépérissement des résineux. Sur le site du Louschbach, pour toutes conditions égales par ailleurs, le dépérissement augmente lorsque l'hydrothermalisme (essentiellement à quartz-hématite) est plus intense.

À l'échelle du site, cette relation peut être également mise en évidence même si les autres facteurs ne sont pas toujours constants. Sur la carte du dépérissement établie par l'IGN à partir de photos aériennes prises en août 1990 (figs 5 et 6), un alignement de placettes très fortement dépérissantes $(\mathrm{N}-\mathrm{S})$ se superpose à la cartographie du filon de quartz.

Les gneiss de la Croix-aux-Mines (fig 2) sont décalés par des failles oủ précipitent micas blancs et quartz. Au niveau d'une petite carrière en bordure de chemin (point 21), la limite entre le granite du Valtin et le gneiss apparaît clairement. La faille est

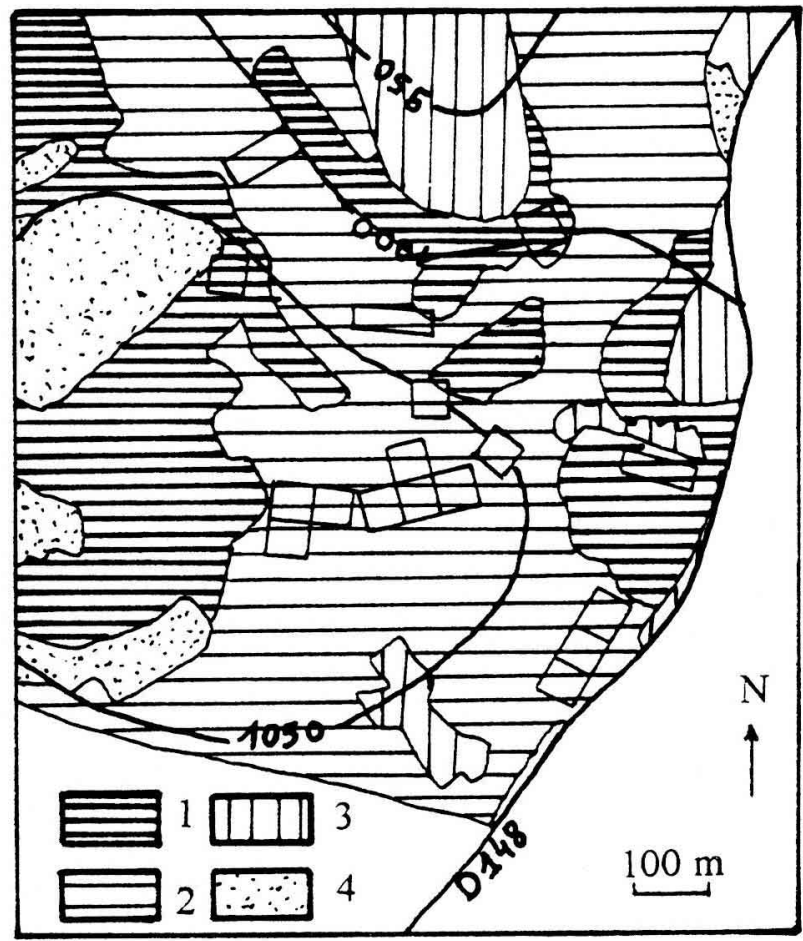

Fig 5. Extrait de la carte du dépérissement établie en août 1990 par l'IGN et positionnement des placettes étudiées sur le site du Louschbach. 1 : zone fortement dépérissante. 2 : zones moyennement dépérissantes. 3 : zones dépérissantes. 4 : zones sans arbres. 


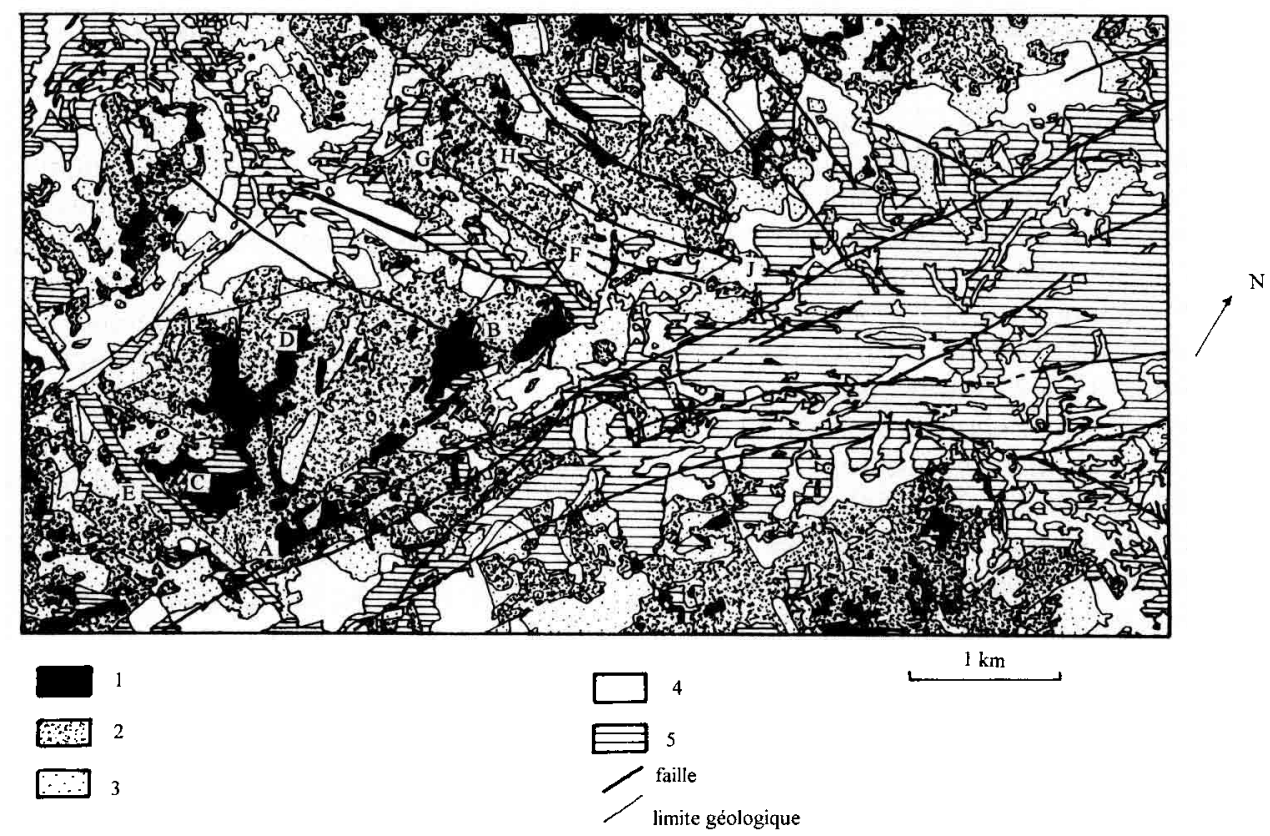

Fig 6. Carte du dépérissement d'après la carte établie par l'IGN (1990) où sont superposées les failles et les limites géologiques d'après la carte géologique au $1 / 50000$ e. $1:$ zone très dépérissante. $2:$ zone moyennement dépérissante. $3:$ zone peu dépérissante. $4:$ zone non dépérissante. $5:$ zone sans arbres. Alignement $\mathrm{AB}$ correspondant au filon de quartz. $\mathrm{C}:$ zone de dépôts de gros blocs glaciaires. $D$ : position de l'affleurement de granite sain GR7. E : dépôts glaciaires au niveau du Sapin de la Vierge. Les segments $F-G$ et $H-J$ correspondent au passage de failles où le dépérissement est très faible. $\mathrm{F}$ se situe au niveau du point 21 (remplissage à mica blanc-quartz).

marquée par la précipitation de micas blancs et de quartz et de rares veinules à hématite. Sur une auréole d'une quinzaine de mètres, le granite est intensément affecté par une altération à micas blancs (biotite et plagioclase entièrement transformés en micas blancs). Sur la carte de I'IGN (fig 6), le passage de ces failles reprises par de l'hydrothermalisme à micas blancs est marqué par des zones peu dépérissantes. Ceci doit être en relation avec l'hydrothermalisme à micas blancs développé aux épontes. Bien que le mica blanc contienne peu de magnésium (environ $1 \%$ de $\mathrm{MgO}$ ), il semble que ce soit suffisant pour éviter un fort dépérissement. Le mica blanc est un minéral peu altérable qui se dissout ou se transforme en vermiculite uniquement dans les horizons du sol. Donc, le potentiel nutritif en magnésium du mica blanc peut être entièrement utilisé lors de leur altération dans le sol.

Une zone fortement dépérissante, située au nord ouest du Louschbach (fig 6), n'est pas en relation avec l'hydrothermalisme de la roche mère. Au niveau de l'affleurement de roche saine (GR7), les peuplements sont dépérissants. D'autres facteurs interviennent : ce sont notamment dans cette zone les dépôts glaciaires. 


\section{Dépôts glaciaires}

Au niveau du mont Louschbach et à l'ouest, des dépôts glaciaires sont observables. Au sommet, de nombreux blocs arrondis décimétriques de granite peu hydrothermalisé et de microganite jalonnent la surface. Actuellement la forêt est absente à cet endroit. En bordure, les arbres sont fortement dépérissants et atteints par des chablis. D'après la carte de I'IGN, cette zone était en 1990 pour moitié boisée par des sapins fortement dépérissants. À cet endroit, sur les dépôts glaciaires se développe un peuplement fort dépérissant. Bonneau et Fichter (1991) ont également remarqué que le dépérissement est généralement plus accentué sur les dépôts périglaciaires.

Un peu plus au sud, au niveau du Sapin de la Vierge, les dépôts glaciaires sont composés de cailloux de granite présentant des degrés d'hydrothermalisme divers. Autant qu'on puisse en juger en se basant sur les cailloux de surface et ceux dégagés par les chablis, la quantité de granite très hydrothermalisé est très hétérogène et peut varier à l'échelle de quelques mètres. Les dépôts glaciaires sont également constitués de grains plus fins non identifiables sur le terrain. II n'a pas été possible de démontrer que les zones plus riches en granite hydrothermalisé sont plus dépérissantes. En revanche, l'intensité du dépérissement est variable à l'échelle de la dizaine de mètres. La carte établie par l'IGN présente sur ces dépôts des zones moyennement dépérissantes et des zones peu dépérissantes.

Manifestement, en présence de dépôts glaciaires, la fertilité du sol est indépendante de la richesse en cations basiques du granite en place. La nature du sol dépend de ces dépôts qui sont souvent très hétérogènes.

\section{Autres facteurs mis en évidence par cette étude}

Les essences sapin et épicéa répondent différemment sur un même sol. Le jaunissement est toujours inférieur sur les sapins. Dans les conditions de roche mère très défavorable, jaunissement et défoliation sont nettement plus marqués sur l'épicéa.

L'altitude, facteur clairement mis en évidence par la carte IGN (rapport IGN, 1991 ; Bonneau et Fichter, 1991), apparaît plus discrètement dans cette étude où ce facteur varie de $60 \mathrm{~m}$ maximum. Par exemple, la placette 15 (1 $030 \mathrm{~m}$ d'altitude), bien que située sur un granite plus hydrothermalisé, présente un état de dépérissement semblable à la placette 6 à $1050 \mathrm{~m}$ d'altitude.

\section{Augmentation artificielle du facteur saturation des sols en cations basiques}

Sur le site du Louschbach, plusieurs essais de fertilisation ont été faits notamment $\mathrm{Ca}$, $\mathrm{Mg}, \mathrm{CaMg}$, NPKCaMg et KMg (fig 3). Après 3 ans, la quantité de calcium et de magnésium échangeables a nettement augmenté (Mohamed Ahmed, 1992). Bonneau (1993) montre que le jaunissement a disparu sur les placettes traitées au calcium et au magnésium, a fortement régressé sur la placette amendée en calcium uniquement et a diminué de moitié sur la placette traitée avec $\mathrm{K}$ et $\mathrm{Mg}$. La masse foliaire a également augmenté après fertilisation. L'augmentation par amendement de la quantité de cations basiques diminue très nettement le dépérissement.

\section{Évolution de la masse foliaire et du jaunissement depuis 1986}

Sur 25 épicéas de la placette T1, des mesures de jaunissement et de défoliation 


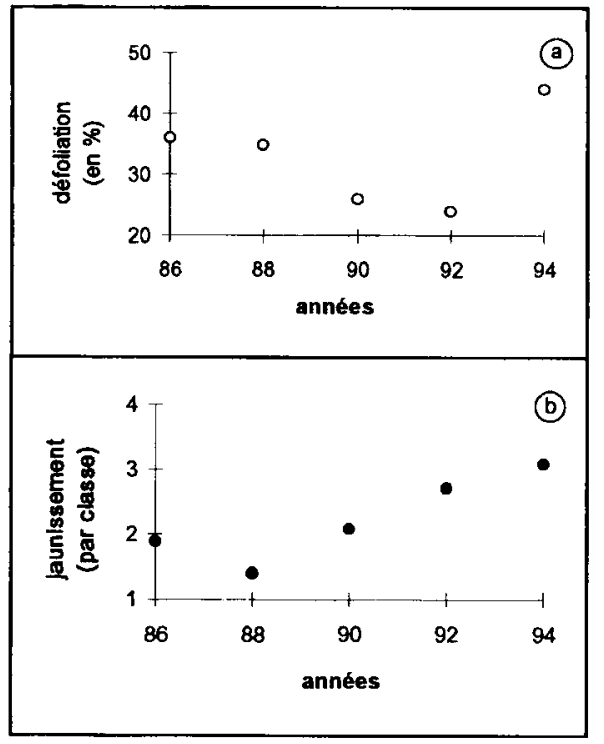

Fig 7. Pourcentage de défoliation a) et jaunissement établi par classe b) du témoin T1 (25 épicéas) durant la période 1986-1994.

ont été pratiquées tous les 2 ans depuis 1986. La figure 7 montre que, depuis 1986 , le jaunissement a baissé en 1988 et augmente constamment depuis lors. La défoliation présente un minimum en 1990-1992. Sur 9 années, défoliation et jaunissement sont les plus accentués en 1994. Sur 8 placettes témoins de résineux dispersées dans les Vosges, Bonneau (1993) constate une diminution de la défoliation et du jaunissement entre 1986 et 1990. Manifestement, en se basant sur la placette T1, la tendance est inversée en 1994 de façon brutale pour la défoliation (+20\% entre 1992 et 1994). Les résultats d'une seule placette ne peuvent pas permettre de conclure à l'échelle des Vosges. En revanche, il est clair que le paramètre temps est important. La figure 8 montre la teneur des aiguilles de l'année courante en magnésium varie inversement au jaunissement entre les années 1985 et

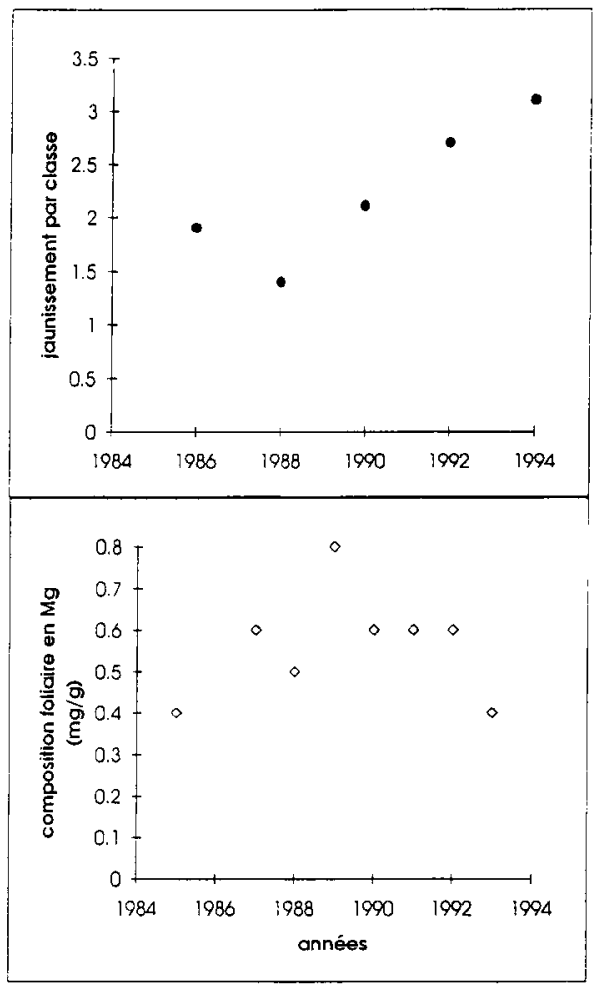

Fig 8. Évolution de la teneur en magnésium des aiguilles de l'année courante et de leur jaunissement établi en classe durant la période 1985 1994.

1994. La teneur maximale est en 1989 avec $0,76 \mathrm{mg}$ de $\mathrm{Mg}$ pour $100 \mathrm{~g}$ de matière sèche.

\section{CONCLUSION}

L'exemple du Louschbach montre clairement que l'un des facteurs jouant sur le dépérissement est la quantité d'éléments nutritifs contenus dans la roche mère. La roche mère ne doit pas être considérée comme ayant la composition de la roche lors de sa mise en place, ce qui est le plus 
souvent donné dans les notices géologiques. Elle peut être localement soumise à des événements hydrothermaux qui modifient sa composition chimique et minéralogique. Au Louschbach, cette transformation a entraîné une diminution importante de la quantité de cations basiques $(-50 \%$ pour $\mathrm{CaO}$ et $-70 \%$ pour $\mathrm{MgO}$ ) qui diminue le potentiel nutritif du granite. De plus, les éléments nutritifs ne sont plus dans les mêmes minéraux, donc ils vont être libérés différemment lors de l'altération supergène des minéraux. De nombreuses paragenèses hydrothermales existent, toutes ne diminuent pas le potentiel nutritif de la roche. De même, cette étude montre que les remplissages des filons sont très variés. Ils ont des effets négatifs sur le potentiel nutritif lorsqu'ils sont remplis de quartz, mais peuvent avoir des effets positifs lorsqu'ils contiennent du mica blanc.

Cette hétérogénéité de composition chimique de la roche mère due à l'hydrothermalisme doit être prise en compte pour des échelles inférieures à 1/50 000 et peut expliquer souvent les variations de dépérissement à l'intérieur d'un même massif géologique pour des conditions topographiques équivalentes.

Les dépôts glaciaires perturbent complètement les conclusions qui sont établies à partir des données sur la roche en place. Au Louschbach, ils sont composés de granite ayant un degré d'hydrothermalisme variable. II est impossible, par une simple cartographie de terrain, d'établir une composition moyenne. Le degré de dépérissement sur ces dépôts varie à l'échelle de quelques dizaines de mètres.

Dans cette étude, en dehors du facteur quantité de cations basiques, pris en compte de manière indirecte, d'autres facteurs comme l'altitude, l'espèce de résineux et le temps ont été mis en évidence. Pour un même degré d'hydrothermalisme, le dépérissement est moins accentué aux altitudes plus faibles. Sur le site du Lou- schbach, les épicéas sont toujours plus jaunes que les sapins dans les mêmes conditions et le plus souvent plus défoliés. Les variations du dépérissement au cours des années sont importants. La défoliation des épicéas de la placette T1 du site du Louschbach a doublé entre l'année 1992 et l'année 1994.

Le facteur quantité de cations basiques est pris en compte indirectement, en considérant qu'il existe une relation entre cette quantité réellement présente sur la capacité d'échange et la quantité potentielle dans la roche mère. L'avantage sur le terrain est qu'une fois calée par une étude précise la roche mère hydrothermalisée peut être cartographiée à l'échelle de la placette forestière et se situe autour de grands filons ou fractures. II ne serait donc plus nécessaire de faire des séries d'analyses d'ions échangeables (au minimum, 10 par placette) pour avoir une idée relative de la variation de cations basiques. Cependant, cette relation doit être confirmée et une étude de l'altération supergène est en cours sur ce site (Turpault et al, 1994).

\section{RÉFÉRENCES}

Belkacem S (1993) Étude de la resaturation des sols acides soumis à de forts apports acides : effet des formes et doses d'amendements sur le fonctionnement d'un sol acide forestier. Thèse univ, Nancy I, $130 \mathrm{p}$

Bonhomme M (1965) Âge, par la méthode au strontium, de quelques granites des Vosges moyennes. Coll Intern Géochr Sc de la Terre Nancy, 10, 3-4, 1-173

Bonneau M, Landmann G, Nys C (1991) Fertilization of declining conifer stands in the Vosges and in the french Ardennes. Water Air Soil Pollut 54, 577-594

Bonneau M, Landmann G (1993) Na Pollution atmosphérique et dépérissement des forêts dans les montagnes françaises. Programme DEFORPA. Rapport 1992, Nancy, INRA, 365 p

Bonneau M (1993) Fertilisation sur résineux adultes (Picea abies Karst et Abies alba Mill) dans les Vosges : composition foliaire en relation avec la défoliation et le jaunissement. Ann Sci For 50, 159175 
Dahire M (1988) Granites et leucogranite péralumineux du Brézouard et du Bilstein (Vosges moyennes) : caractères pétrographiques, géochimiques et minéralogiques. Thèse univ, Nancy i, $158 p$

Fluck $P$ (1980) Métamorphisme et magmatisme dans les Vosges moyennes d'Alsace. Contribution à l'histoire de la chaîne varisque. Sci Géol 62, 248 p

Griffault L (1987) Bilan des transferts de matière (majeurs et terres rares) au cours des altérations hydrothermales des granites. Exemple du granite du ballon d'Alsace (Vosges méridionales). Thèse univ, Poitiers, $134 \mathrm{p}$

Hameurt (1967) Les terrains cristallins et cristallophylliens du versant occidental des Vosges moyennes. Mém Sen Carte Géol Als Lorr 26, $402 \mathrm{p}$

Landmann $G$ (1991) French research into forest decline. DEFORPA Programme, 2nd report, ENGREF, Nancy, $120 \mathrm{p}$

Landmann G (1992) Research into forest decline and air pollution in France. Major findings and relevance for policy applications. In : Acidification Research, Evaluation and Policy Applications (T Schneider, ed), Elsevier, Amsterdam, 81-92

Mohamed Ahamed D (1992) Rôle du facteur édaphique dans le fonctionnement biogeochimique de 2 pessières vosgiennes : effet d'un amendement calcimagnésien. Thèse univ, Nancy $1,136 \mathrm{p}$

Mohamed Ahamed D, Ranger J, Dambrienne E, Bonneau M, Gelhaye D, Granier A (1993) The effects of limestone and of limestone plus NPK fertilization on the soil and mass-balance of a spruce stand
(Picea abies (L) Karst) in the Vosges mountains. Forest Ecol Manag 60, 291-310

Nys C (1989) Fertilisation, dépérissement et production de l'épicéa commun (Picea abies Karst) dans les Ardennes. Rev For Fr 4, 336-347

Pagel M (1981) Facteurs de distribution et de concentration de l'uranium et du thorium dans quelques granites de la chaîne hercynienne d'Europe. Thèse d'État, Nancy I

Ranger J, Mohammed Ahamed D, Gelhaye D (1994) Effet d'un amendement calco-magnésien associé ou non à une fertilisation, sur le cycle biogéochimique des éléments nutritifs dans une plantation d'épicéa commun (Picea abies Karst) dépérissante dans les Vosges. Ann Sci For 51, 455-475

Rapport IGN (1991) Dépérissement des forêts du col du Bonhomme (Vosges, haut Rhin), 21 p

Samuel J, Rouault R (1983) Les méthodes d'analyses des matériaux géologiques pratiquée au laboratoire d'analyses spectrochimiques. Notes techniques de l'institut de géologie, $n^{\circ} 16$, université Louis-Pasteur, Strasbourg

Turpault MP, Bonnaud P, Fichter J, Ranger J (1994) Profil d'altération développé sur un granite affecté par l'hydrothermalisme à quartz et hématite (Louschbach, Vosges). Bull Liaison Soc Fr Minéral Cristallogr 6, 34

Turpault MP, Berger G, Meunier A (1992) Dissolutionprecipitation processes induced by hot water in a fractured granite. Part 1. Wall-rock alteration and vein deposition processes. Eur J Minera/ 4, 1457-1475 